\title{
SGLT2 inhibition and ketoacidosis - should we be concerned?
}

\author{
SURYA PANICKER RAJEEV, JOHN PH WILDING
}

\begin{abstract}
SGLT2 inhibitors represent a novel class of oral glucoselowering treatment that addresses some important unmet clinical needs in the treatment of type 2 diabetes, specifically weight reduction and a low propensity to cause hypoglycaemia. SGLT2 inhibition lowers the renal threshold for glucose excretion, resulting in renal glycosuria, a shift in substrate utilisation from carbohydrate to fat oxidation and hyperglucagonaemia; this poses a theoretical risk for ketoacidosis (including euglycaemic ketoacidosis) in the presence of other precipitating factors, especially reduction in insulin doses or low carbohydrate intake. There have been reports of several cases of ketoacidosis, mostly euglycaemic, and in people with type 1 or type 2 diabetes. Subsequent to this there were warnings from regulatory bodies (FDA and EMEA). In this article, we examine the reports of ketoacidosis associated with SGLT2 inhibition and try to explain the intrinsic pathophysiological mechanisms associated with this class of drugs which might contribute to ketoacidosis. The implications of these for clinical practice are summarised with key messages to health care providers.

Br J Diabetes Vasc Dis 2015;15:155-158
\end{abstract}

Key words: SGLT2 inhibitors, ketoacidosis, type 2 diabetes, mechanisms, clinical practice

\section{Background}

SGLT2 inhibitors are a new class of drugs for the treatment of type 2 diabetes that act by inhibiting renal glucose reabsorption. They have been adopted rapidly into clinical practice guidelines due to a combination of glucose lowering with weight reduction. ${ }^{1-3}$ The difficulty in matching the normal physiology of insulin secretion with exogenous insulin use is a constant challenge when managing type 1 diabetes. As weight gain is a frequent consequence of intensive glucose control, ${ }^{4}$ the use of adjunctive treatments that

Obesity and Endocrinology Research Group, Institute of Ageing and Chronic Disease, University of Liverpool

Address for correspondence: Professor John PH Wilding Obesity and Endocrinology Research Group, University of Liverpool, Clinical Sciences Centre, Aintree University Hospital NHS Foundation Trust, Longmoor Lane, Liverpool, L9 7AL, UK.

Tel: +44 (0)1515295899

E-mail: J.P.H.Wilding@liverpool.ac.uk

http://dx.doi.org/10.15277/bjdvd.2015.047 promote weight loss is sometimes considered. In addition, there is preliminary evidence that SGLT2 inhibitors may attenuate the progression of kidney disease in type 1 diabetes by decreasing glomerular hyperfiltration. 5,6 As a result of these preliminary data, some clinicians have considered SGLT2 inhibitors to represent an attractive option in type 1 diabetes, resulting in off-label use in this population. Case reports of diabetic ketoacidosis (DKA) associated with SGLT2 inhibitors started to appear earlier this year, 7,8 subsequent to reports of DKA in patients with type 1 diabetes in clinical trials. 5,9 The US Food and Drug Administration (FDA) published a formal warning regarding this potential complication in May 2015, reporting cases not only in type 1 diabetes, but also in type 2 diabetes. ${ }^{10}$ This has been followed by a similar warning from the European Medicines Evaluation Agency (EMEA) ${ }^{11}$ and the manufacturers of the three currently approved SGLT2 inhibitors, dapagliflozin, canagliflozin and empagliflozin.

In this article we review the evidence behind this potential risk associated with SGLT2 inhibitors, the likely contributory mechanisms for ketoacidosis and the clinical implications for practising physicians and patients.

\section{The magnitude of the problem}

The FDA warning regarding this potential complication was based on 20 cases of ketoacidosis in patients taking SGLT2 inhibitors identified from the FDA Adverse Event Reporting System database between 2013 and 2014. ${ }^{10}$ Although most of these patients had type 2 diabetes, details of these cases and other contributory factors are not known. Subsequently, nine patients on SGLT2 inhibitors (seven with type 1 diabetes and two with type 2 diabetes) with 13 episodes of ketoacidosis were described in a case series. ${ }^{12}$ Interestingly, all presented with euglycaemic DKA (euDKA) that led to a delay in diagnosis. A further report described two cases of euDKA in SGLT2 inhibitortreated patients, both with misdiagnosed secondary diabetes. ${ }^{13}$

From the limited available data, the risk appears to be firstly of euDKA in type 1 diabetes and in the context of pancreatic ( $\beta$ cell) failure. SGLT2 inhibitors were shown to have beneficial effects on glucose profiles with lower daily insulin doses in type 1 diabetes from short term studies ${ }^{9,14}$; nevertheless, worries regarding ketosis were noted. ${ }^{15}$ Recognised precipitating factors for ketoacidosis were noted in the two patients with type 2 diabetes who developed ketoacidosis: poor nutrition and postoperative stress. The blood glucose levels of reported patients range from $5.3-12.9 \mathrm{mmol} / \mathrm{L}$ and the lowering of renal threshold of glucose excretion in the context of increased free fatty acid generation was the postulated mechanism for euDKA. ${ }^{16}$ The 
EMEA has retrieved 147 cases of ketoacidosis reported worldwide through a search in the EudraVigilance (EV) database: 96 cases with canagliflozin, 46 cases with dapagliflozin and 7 cases with empagliflozin. Of these, 46 patients had a diagnosis of type 1 diabetes. ${ }^{11}$ The background rate of DKA in type 2 diabetes has been reported to be 0.5 cases per 1000 patient-years ${ }^{17}$ and the estimated exposure to SGLT2 inhibitors is over half a million patient-years. This is most likely a class effect; most of the published cases were associated with canagliflozin, but this probably relates to the more widespread use of this agent in the USA due to its longer availability.

More recently, Erondu et al reported the incidence of DKA in 17,596 patients from randomised control studies of canagliflozin up until May 2015 which were 0.522 per 1000 patient-years (canagliflozin $100 \mathrm{mg}$ ), 0.763 per 1000 patient-years (canagliflozin $300 \mathrm{mg}$ ) and 0.238 per 1000 patient-years (comparators). ${ }^{18}$ The cardiovascular outcomes trial for empagliflozin in 7,028 patients (EMPA-REG) reported an incidence of DKA of $<0.1 \%$ with no difference between empagliflozin and placebo groups. $^{19}$

\section{Mechanism of ketoacidosis with SGLT2 inhibition}

The triad of hyperglycaemia (blood glucose $>11 \mathrm{mmol} / \mathrm{L}$ ), acidosis (venous $\mathrm{HCO}_{3}{ }^{-}<15 \mathrm{mmol} / \mathrm{L}$ ) and ketonaemia or ketonuria associated with DKA occurs in the context of insulin deficiency and counter-regulatory hormone excess with possible contributory precipitating factors like stress, illness or omission of insulin. EuDKA is the combination of metabolic acidosis and ketosis in the context of normoglycaemia and is often perpetuated by contributory factors such as poor nutrition or alcohol intake. It is possible to maintain normoglycaemia even in a state of severe and absolute insulin dearth by augmenting renal glycosuria. Hyperglycaemia usually precedes ketoacidosis in type 1 diabetes and the normoglycaemic state in the context of ketoacidosis may cause delay in the diagnosis.

Although the reports so far suggest most of the cases were associated with one or more contributory factors, there are intrinsic metabolic effects of SGLT2 inhibitors which can compound this problem. Tofogliflozin has been demonstrated to cause a dose-dependent hyperketonaemia and ketonuria in a combined phase 2 and 3 trial in patients with type 2 diabetes, though none of the patients required emergency admission. ${ }^{20}$ Data from rodent models showed that SGLT2 inhibition can cause an increase in $\beta$-hydroxybutyric acid (BHBA) levels; this finding was seen in the fasting state and in a calorie-restricted group in the non-fasting state. ${ }^{21}$ Ipragliflozin was also demonstrated to have similar effects on plasma and urinary BHBA levels as well as plasma levels of non-esterified fatty acids (NEFA) in the fasted state, implying augmentation of fatty acid oxidation and lipolysis, respectively.22 Since SGLT2 inhibition promotes lipolysis, the resulting increase in FFA levels contributes to ketogenesis. This is even more important in states of relative insulin deficiency or low carbohydrate intake.

There is increased $\mathrm{Na}^{+}$concentration in renal tubular fluid as a result of decreased Na reabsorption due to SGLT2 inhibition. The resultant increase in positive electrical charge in the tubular
Table 1 Predisposing factors for DKA in patients on SGLT2 inhibitors

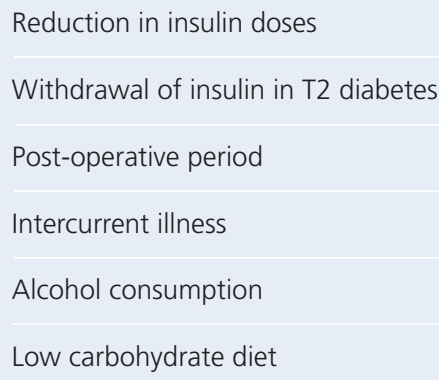

lumen due to the $\mathrm{Na}^{+}$ions may drive the ketone bodies (which are negatively charged) into tubular fluid resulting in reduced clearance of urinary ketones. This leads to elevation of plasma ketone levels. ${ }^{23}$ This could pose a problem (in conjunction with normoglycaemia) in early recognition of ketoacidosis in patients who monitor urine ketones.

This effect was noted in animal studies of phlorizin, the prototype non-specific SGLT inhibitor. ${ }^{24}$ Phlorizin increases plasma acetoacetate and 3-hydroxy butyrate levels during fasting in animal models. ${ }^{25}$ It has also been postulated that the sodiummonocarboxylate transporter 1, SLC5A8, in the epithelial cells of renal tubules may be involved in the co-transport of ketone bodies with $\mathrm{Na}^{+}$, and SGLT2 inhibition might have similar effects, resulting in decreased renal clearance of ketone bodies..$^{23}$

Hyperglucagonaemia, a recently observed 26,27 and explained ${ }^{28}$ metabolic effect of SGLT2 inhibition could augment hepatic ketogenesis. ${ }^{29}$ Animal studies suggested this effect might be due to increased kisspeptin-1 secretion in the liver. ${ }^{30}$ SGLT2 expression has been demonstrated in $\alpha$-cells of the pancreas and may be a component of the $\alpha$-cell glucose sensing mechanism, as inhibition of this process in human islets results in increased glucagon secretion. ${ }^{28}$ SGLT2 inhibition could also augment the hypovolaemic state associated with DKA due to the diuresis of around $400 \mathrm{~mL} /$ day. This in turn could increase the secretion of counter-regulatory hormones resulting in lipolysis and ketogenesis.

During fasting, when insulin concentrations are low, glucose can be maintained at close to normal levels during SGLT2 inhibition, despite glycosuria, due to increased hepatic gluconeogenesis. However, suppression of hepatic ketogenesis requires relatively higher insulin concentrations. SGLT2 inhibition has an intrinsic propensity to increase ketogenesis as it lowers insulin concentrations, increases glucagon secretion, and promotes lipolysis. For the majority of patients with type 2 diabetes, SGLT2 inhibition helps lower blood glucose and reduces body fat. SLGT2 inhibition augments the hepatic ketogenesis pathway in patients with type 1 diabetes, secondary diabetes due to pancreatic insufficiency, in type 2 diabetes in the context of severe metabolic stress (e.g. sepsis or after major surgery), or a reduction of exogenous insulin or restricted carbohydrate intake.

Available reports included contributory factors which led to DKA, like poor nutrition, post-surgery and associated stress, 
reduction in insulin doses related to glycaemic improvement or an adaptation to lifestyle. However, the contribution of the above explained metabolic processes secondary to SGLT2 inhibition should not be overlooked. Table 1 summarises some of the factors associated with precipitation of DKA in patients treated with SGLT2 inhibitors.

\section{Implications for practice \\ Type 1 diabetes}

Short-term trial data in a small number of type 1 diabetes patients have suggested that SGLT2 inhibition may improve glycaemic profiles, help with weight loss, and potentially reduce the progression of diabetic renal disease. However, euDKA was observed during at least one of these trials, 5,9 and SGLT2 inhibitors are not currently approved for use in patients with type 1 diabetes. Hence, it is prudent to avoid the unlicensed use of this class of drugs in the light of these warnings, at least until more safety data from randomised control trials are available.

Patients need to be counselled regarding the potential risk of ketoacidosis, symptoms of DKA (reinforced with special emphasis on euDKA) when a SGLT2 inhibitor is given in a research setting. Monitoring of ketones in blood or urine is essential. In the presence of ketonuria or ketosis, advice should be given to withhold the SGLT2 inhibitor, maintain carbohydrate and fluid intake to allow continuation of insulin therapy at optimal doses and to seek medical attention depending on the severity.

\section{Type 2 diabetes}

In the context of type 2 diabetes, physicians need to be aware of euDKA with use of SGLT2 inhibitors, especially at times of poor nutrition or stress following surgery. The intriguing metabolic effects of SGLT2 inhibition have not been fully elucidated and the metabolic stress in the post-operative period may augment these effects. Until more information is available, it might be prudent to withhold SGLT2 inhibitors for a longer duration than its therapeutic half-life during the peri-operative period. Providers also need to be cautious of the propensity for ketoacidosis in relation to alcohol intake (although reports suggested not more than usual average intake) as well as reduction in insulin doses secondary to glycaemic improvement or in relation to physical activity. However, we do not think there is enough evidence from available data to recommend regular monitoring of ketones in patients with type 2 diabetes taking a SGLT2 inhibitor.

All reported cases responded to standard treatment for DKA with intravenous fluids, electrolyte supplementation and insulin infusion.

\section{Cautions}

It would be prudent to advise patients to withhold SGLT2 inhibitors temporarily if they cannot maintain adequate fluid intake, due to their propensity to worsen the hypovolaemic state. Patients should not be re-challenged immediately with SGLT2 inhibitor treatment after episodes of ketoacidosis, as this can cause recurrence of their metabolic derangement. ${ }^{12}$ Accurate

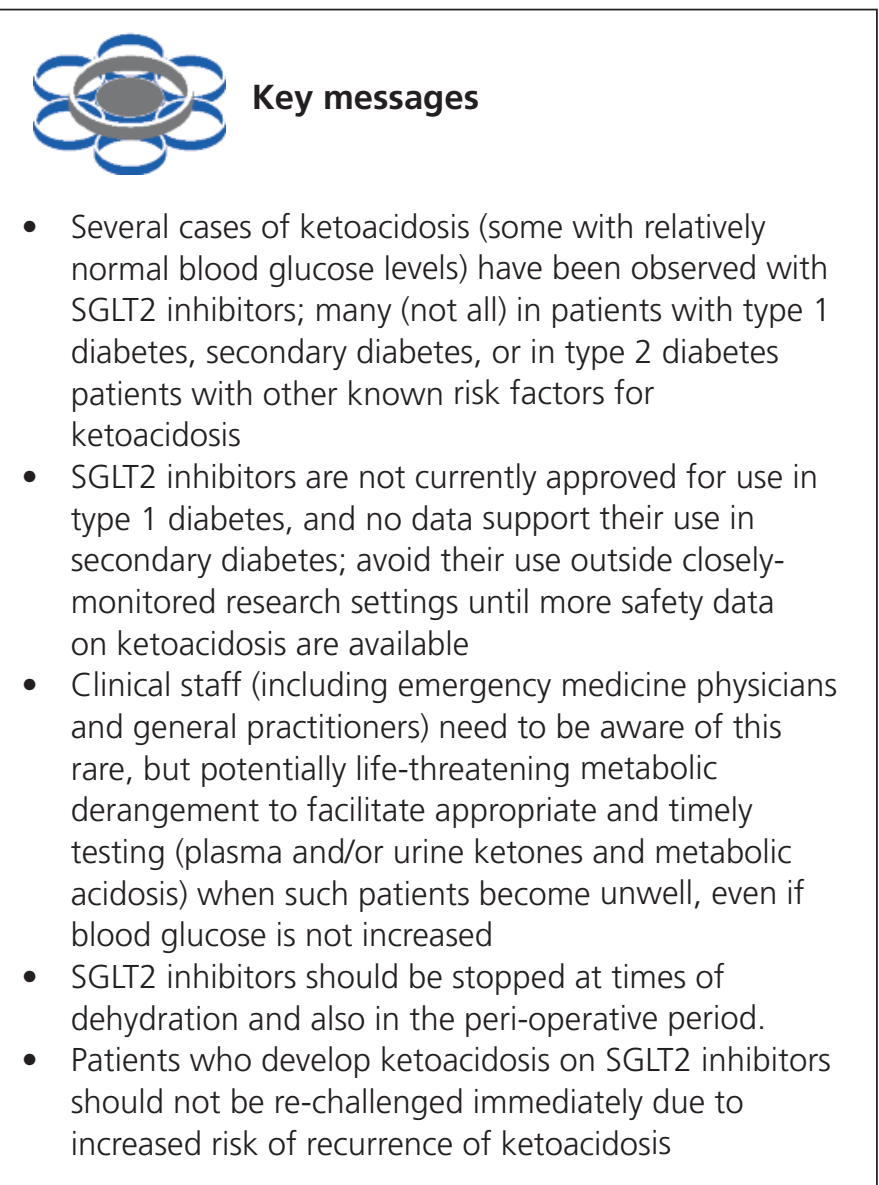

diagnosis of type 2 diabetes is essential as episodes of euDKA were observed in patients with secondary diabetes due to pancreatic damage who were incorrectly diagnosed as having type 2 diabetes.

\section{Summary}

SGLT2 inhibitors, the latest class of glucose-lowering drugs, facilitate lowering of hyperglycaemia by inhibiting renal glucose re-absorption and promoting glycosuria. When used appropriately in type 2 diabetes (their licensed indication), they display therapeutic advantages compared to some other glucose lowering drugs, particularly a reduction in body weight secondary to energy loss, a low intrinsic risk of hypoglycaemia and a moderate reduction in systolic blood pressure.

SGLT2 inhibitors are currently being investigated for use in type 1 diabetes. Short-term results have demonstrated glycaemic efficacy, reduction in insulin doses and, more notably, a reduction in intra-glomerular pressures resulting in reversal of glomerular hyperfiltration, one of the pathologies associated with the development of diabetic kidney disease. However, the side effect profile of SGLT2 inhibitors should be carefully studied before extrapolating these data to clinical practice, especially in the context of reports of ketoacidosis.

SGLT2 inhibitors may be associated with ketoacidosis due to their ketogenic effects secondary to enhanced lipolysis and hyperglucagonaemia and this could pose a problem in the presence of 
other contributory factors for ketoacidosis. Nevertheless, this is an association and a cause-effect relationship has not been proven to date. Hence, in the context of type 2 diabetes relevant to UK practice, there is insufficient evidence for serious alarm, though we urge physicians to be aware of the susceptibility of patients to develop this metabolic complication, especially with other predisposing factors. We also need more safety data from large randomised, controlled trials regarding SGLT2 inhibitor use in type 1 diabetes and type 2 diabetes in the peri- and post-operative states.

Conflict of interest JPHW has acted as a consultant, received institutional grants and given lectures on behalf of pharmaceutical companies developing or marketing medicines used for the treatment of obesity and diabetes, including Boehringer-Ingelheim (manufacturer of empagliflozin), AstraZeneca and Janssen (manufacturers of dapagliflozin and canagliflozin respectively). SPR has no conflicts of interest.

\section{Funding None.}

\section{References}

1. NICE Technology Appraisal Guidance (TA288). Dapagliflozin in combination therapy for treating type 2 diabetes. 2013. Available at https://www.nice.org.uk/guidance/ta288.

2. Inzucchi SE, Bergenstal RM, Buse JB, et al. Management of hyperglycemia in type 2 diabetes, 2015: a patient-centered approach: update to a position statement of the American Diabetes Association and the European Association for the Study of Diabetes. Diabetes Care 2015; 38:140-9. http://dx.doi.org/10.2337/dc14-2441

3. Handelsman Y, Bloomgarden ZT, Grunberger G, et al. American Association of Clinical Endocrinologists and American College of Endocrinology - clinical practice guidelines for developing a diabetes mellitus comprehensive care plan - 2015. Endocrine Pract 2015:21 Suppl 1:1-87. http://dx.doi.org/10.4158/EP15672.GLSUPPL

4. The Diabetes Control and Complications Trial Research Group. The effect of intensive treatment of diabetes on the development and progression of long-term complications in insulin-dependent diabetes mellitus. New Engl J Med 1993;329:977-86.

5. Cherney DZI, Perkins BA, Soleymanlou N, et al. Renal hemodynamic effect of sodium-glucose cotransporter 2 inhibition in patients with type 1 diabetes mellitus. Circulation 2014:129:587. http://dx.doi.org/10.1161/CIRCULATIONAHA.113.005081

6. Skrtic M, Yang GK, Perkins BA, et al. Characterisation of glomerular haemodynamic responses to SGLT2 inhibition in patients with type 1 diabetes and renal hyperfiltration. Diabetologia 2014;57:2599-602. http://dx.doi.org/10.1007/s00125-014-3396-4

7. St Hilaire R, Costello H. Prescriber beware: report of adverse effect of sodium-glucose cotransporter 2 inhibitor use in a patient with contraindication. Am J Emerg Med 2015;33:604.e3-4. http://dx.doi.org/10.1016/j.ajem.2014.09.039

8. Hayami T, Kato $\mathrm{Y}, \mathrm{Kamiya} \mathrm{H}$, et al. Case of ketoacidosis by a sodiumglucose cotransporter 2 inhibitor in a diabetic patient with a low-carbohydrate diet. J Diabetes Invest 2015;6:587-90. http://dx.doi.org/10.1111/jdi.12330

9. Perkins BA, Cherney DZ, Partridge $H$, et al. Sodium-glucose cotransporter 2 inhibition and glycemic control in type 1 diabetes: results of an 8-week open-label proof-of-concept trial. Diabetes Care 2014;37:1480-3. http://dx.doi.org/10.2337/dc13-2338

10. FDA Drug Safety Communication:FDA warns that SGLT2 inhibitors for diabetes may result in a serious condition of too much acid in the blood. Available from http://www.fda.gov/Drugs/DrugSafety/ucm446845.htm DOI:15-05-2015.

11. European Medicines Agency. Review of diabetes medicines called SGLT2 inhibitors started. Risk of diabetic ketoacidosis to be examined. Available from http://www.ema.europa.eu/ema/index.jsp?curl=pages/medicines/ human/referrals/SGLT2_inhibitors/human_referral_prac_000052.jsp\&mid
$=$ WC0b01ac05805c516f.

12. Peters AL, Buschur EO, Buse JB, et al. Euglycemic diabetic ketoacidosis: a potential complication of treatment with sodium-glucose cotransporter 2 inhibition. Diabetes Care 2015;38:1687-93. http://dx.doi.org/10.2337/dc15-0843

13. Hine J, Paterson H, Abrol E, et al. SGLT inhibition and euglycaemic diabetic ketoacidosis. Lancet Diabetes Endocrinol 2015;3:503-04. http://dx.doi.org/10.1016/S2213-8587(15)00204-1

14. Henry RR, Rosenstock J, Edelman $S$, et al. Exploring the potential of the SGLT2 inhibitor dapagliflozin in type 1 diabetes: a randomized, doubleblind, placebo-controlled pilot study. Diabetes Care 2015;38:412-19. http://dx.doi.org/10.2337/dc13-2955

15. Lamos EM, Younk LM, Davis SN. Empagliflozin, a sodium glucose cotransporter 2 inhibitor, in the treatment of type 1 diabetes. Expert Opin Investig Drugs 2014;23:875-82.

http://dx.doi.org/10.1517/13543784.2014.909407

16. Ireland JT, Thomson WS. Euglycemic diabetic ketoacidosis. BMJ 1973; 3:107. http://dx.doi.org/10.1136/bmj.3.5871.107-a

17. Wang ZH, Kihl-Selstam E, Eriksson JW. Ketoacidosis occurs in both Type 1 and Type 2 diabetes-a population-based study from Northern Sweden. Diabetic Med 2008:25:867-70. http://dx.doi.org/10.1111/j.1464-5491.2008.02461.x

18. Erondu N, Desai M, Ways K, Meininger G. Diabetic ketoacidosis and related events in the canagliflozin type 2 diabetes clinical program. Diabetes Care 2015;38:1680-6. http://dx.doi.org/10.2337/dc15-1251

19. Zinman B, Wanner C, Lachin JM, et al. Empagliflozin, cardiovascular outcomes, and mortality in type 2 diabetes. N Engl J Med 2015 (in press) http://dx.doi.org/10.1056/NEJMoa1504720

20. Kaku K, Watada H, Iwamoto Y, et al. Efficacy and safety of monotherapy with the novel sodium/glucose cotransporter-2 inhibitor tofogliflozin in Japanese patients with type 2 diabetes mellitus: a combined Phase 2 and 3 randomized, placebo-controlled, double-blind, parallel-group comparative study. Cardiovasc Diabetol 2014;13:65. http://dx.doi.org/10.1186/1475-2840-13-65

21. Devenny JJ, Godonis HE, Harvey SJ, et al. Weight loss induced by chronic dapagliflozin treatment is attenuated by compensatory hyperphagia in diet-induced obese (DIO) rats. Obesity (Silver Spring) 2012;20:1645-52. http://dx.doi.org/10.1038/oby.2012.59

22. Yokono M, Takasu T, Hayashizaki Y, et al. SGLT2 selective inhibitor ipragliflozin reduces body fat mass by increasing fatty acid oxidation in high-fat diet-induced obese rats. Eur J Pharmacol 2014;727:66. http://dx.doi.org/10.1016/j.ejphar.2014.01.040

23. Taylor SI, Blau JE, Rother KI. Perspective: SGLT2 inhibitors may predispose to ketoacidosis. J Clin Endocrinol Metab 2015;100:2849-52. http://dx.doi.org/10.1210/jc.2015-1884

24. Cohen JJ, Berglund F, Lotspeich WD. Renal tubular reabsorption of acetoacetate, inorganic sulfate and inorganic phosphate in the dog as affected by glucose and phlorizin. Am J Physiol 1956;184:91-6.

25. Ruderman NB, Ross PS, Berger M, Goodman MN. Regulation of glucose and ketone-body metabolism in brain of anaesthetized rats. Biochem $J$ 1974;138:1-10. http://dx.doi.org/10.1042/bj1380001

26. Ferrannini E, Muscelli E, Frascerra S, et al. Metabolic response to sodiumglucose cotransporter 2 inhibition in type 2 diabetic patients. J Clin Invest 2014;124:499-508. http://dx.doi.org/10.1172/JCI72227

27. Merovci A, Solis-Herrera C, Daniele G, et al. Dapagliflozin improves muscle insulin sensitivity but enhances endogenous glucose production J Clin Invest 2014;124:509-14. http://dx.doi.org/10.1172/JCI70704

28. Bonner C, Kerr-Conte J, Gmyr V, et al. Inhibition of the glucose transporter SGLT2 with dapagliflozin in pancreatic alpha cells triggers glucagon secretion. Nature Med 2015;21:512-17. http://dx.doi.org/10.1038/nm.3828

29. Keller U, Schnell H, Sonnenberg GE, et al. Role of glucagon in enhancing ketone body production in ketotic diabetic man. Diabetes 1983;32:38791. http://dx.doi.org/10.2337/diab.32.5.387

30. Song WJ, Mondal P, Wolfe A, et al. Glucagon regulates hepatic kisspeptin to impair insulin secretion. Cell Metab 2014;19:667-81. http://dx.doi.org/10.1016/j.cmet.2014.03.005 